\title{
Epidemiologia da tuberculose bovina na América do Sul
}

\author{
Epidemiology of bovine tuberculosis in South America \\ Epidemiología de la tuberculosis bovina en Sudamérica
}

Recebido: 06/07/2021 | Revisado: 09/07/2021 | Aceito: 09/07/2021 | Publicado: 21/07/2021

\author{
Murilo da Silva Garcia \\ ORCID: https://orcid.org/0000-0001-5230-9059 \\ Universidade Brasil, Brasil \\ E-mail: murilogarcia492@gmail.com \\ Amanda Freitas Melo \\ ORCID: https://orcid.org/0000-0001-5374-8416 \\ Universidade Brasil, Brasil \\ E-mail: meloamandafreitas@gmail.com \\ Gabriel Faria Carvalho \\ ORCID: https://orcid.org/0000-0001-8649-7588 \\ Universidade Brasil, Brasil \\ E-mail: fariagabrie1970@gmail.com \\ Gabriel Pinheiro Pomim \\ ORCID: https://orcid.org/0000-0002-2962-5093 \\ Universidade Brasil, Brasil \\ E-mail: gabrielpopulina30@gmail.com \\ Pedro Manoel de Souza Neves \\ ORCID: https://orcid.org/0000-0003-4763-5209 \\ Universidade Brasil, Brasil \\ E-mail: pedromanoel103209@gmail.com \\ Richarlla Aparecida Buscariol Silva \\ ORCID: https://orcid.org/0000-0002-3190-8332 \\ Universidade Brasil, Brasil \\ E-mail: buscariolricharlla@gmail.com \\ Rafael Ovídio de Oliveira \\ ORCID: https://orcid.org/0000-0002-1863-877X \\ Secretaria de Estado de Saúde de Mato Grosso do Sul, Brasil \\ E-mail: rafaovidio29@gmail.com \\ Danila Fernanda Rodrigues Frias \\ ORCID: https://orcid.org/0000-0001-8621-3338 \\ Universidade Brasil, Brasil \\ E-mail: danila.frias@universidadebrasil.edu.br
}

\begin{abstract}
Resumo
A presente pesquisa teve por objetivo descrever a epidemiologia dos casos de tuberculose bovina na América do Sul, visando enfatizar a importância das medidas de controle e prevenção da doença. Para esta pesquisa foi realizado um estudo transversal, descritivo, retrospectivo e qualiquantitativo que utilizou como unidades de análise países localizados na América do Sul. A amostra foi delimitada ao período de 2012 a 2018, e os dados foram coletados a partir das informações disponíveis em banco de dados oficiais da Organização Internacional de Epizootias (OIE) e do Ministério da Agricultura, Pecuária e Abastecimento (MAPA). Os resultados foram analisados por meio de estatística descritiva simples. Os países que apresentaram maior número de casos de tuberculose bovina notificados foram Argentina e Chile apresentando prevalência média de $0,46 \%$ e $0,96 \%$, respectivamente. O Brasil apresentou prevalência média de $0,02 \%$, com taxa mais elevada em Santa Catarina $(0,21 \%)$, Distrito Federal $(0,11 \%)$, Rio Grande do Sul $(0,09 \%)$, e Paraná $(0,09 \%)$. Com relação a prevalência de focos, destacou-se os estados do Paraná $(1,35 \%)$, Distrito Federal (1,29\%) e Santa Catarina (0,88\%). Conclui-se que os países da América do Sul, inclusive o Brasil, possuem baixa taxa de prevalência de tuberculose bovina, porém estratégias relacionadas a vigilância da doença devem ser tomadas aliadas a ações de educação em saúde voltadas aos produtores rurais e a população visando minimizar o risco de ocorrência da tuberculose zoonótica.
\end{abstract}

Palavras-chave: Mycobacterium bovis; Saúde única; Tuberculose zoonótica.

\section{Abstract}

Bovine tuberculosis is a zoonosis of chronic evolution, and despite its high prevalence, it is considered a neglected disease. It belongs to the list of diseases notifiable to the World Organisation for Animal Health (OIE), which reinforces its importance as a serious public health problem. In this scenario, this study describes the epidemiology of cases of bovine tuberculosis in South America to emphasize the importance of measures to control and prevent the disease. A cross-sectional, descriptive, retrospective and quali-quantitative investigation was carried out using 
countries located in South America as units of analysis. The sample was delimited to the period from 2012 to 2018 , and data were collected from information available in the official database of the International Organization of Epizootics (Office International des Epizooties, OIE) and the Brazilian Ministry of Agriculture, Livestock and Supply (Ministério da Agricultura, Pecuária e Abastecimento, MAPA). Results were analyzed using simple descriptive statistics. The countries with the highest number of reported cases of bovine tuberculosis were Argentina and Chile, with an average prevalence of $0.46 \%$ and $0.96 \%$, respectively. Brazil had an average prevalence of $0.02 \%$, with the highest rates occurring in the states of Santa Catarina (0.21\%), Federal District $(0.11 \%)$, Rio Grande do Sul $(0.09 \%)$ and Paraná $(0.09 \%)$. In terms of prevalence of outbreaks, the states of Paraná $(1.35 \%)$, Federal District $(1.29 \%)$ and Santa Catarina $(0.88 \%)$ stood out. In conclusion, South American countries, including Brazil, have a low prevalence rate of bovine tuberculosis, but strategies related to the surveillance of the disease must be adopted alongside health education actions aimed at rural producers and the population to minimize the risk of occurrence of this zoonotic tuberculosis.

Keywords: Mycobacterium bovis; One health; Zoonotic tuberculosis.

\section{Resumen}

Esta investigación tuvo como objetivo describir la epidemiología de los casos de tuberculosis bovina en América del Sur, con el objetivo de enfatizar la importancia de las medidas de control y prevención de la enfermedad. Para esta investigación se realizó un estudio transversal, descriptivo, retrospectivo y cualitativo, utilizando como unidades de análisis países ubicados en Sudamérica. La muestra se delimitó al período de 2012 a 2018, y los datos se recolectaron a partir de la información disponible. en la base de datos datos oficiales de la Organización Internacional de Epizootias (OIE) y del Ministerio de Agricultura, Ganadería y Abastecimiento (MAPA). Los resultados se analizaron mediante estadística descriptiva simple. Los países con mayor número de casos notificados de tuberculosis bovina fueron Argentina y Chile, con una prevalencia promedio de 0,46\% y 0,96\%, respectivamente. Brasil tuvo una prevalencia promedio de $0.02 \%$, con la tasa más alta en Santa Catarina (0.21\%), Distrito Federal $(0.11 \%)$, Rio Grande do Sul $(0.09 \%)$ y Paraná $(0,09 \%)$. En cuanto a la prevalencia de brotes, se destacaron los estados de Paraná $(1,35 \%)$, Distrito Federal $(1,29 \%)$ y Santa Catarina (0,88\%). Se concluye que los países de América del Sur, incluido Brasil, tienen una baja tasa de prevalencia de tuberculosis bovina, pero se deben tomar estrategias relacionadas con la vigilancia de la enfermedad junto con acciones de educación en salud dirigidas a los productores rurales y la población con el fin de minimizar el riesgo de aparición de tuberculosis zoonótica.

Palabras clave: Mycobacterium bovis; Salud única; Tuberculosis zoonótica.

\section{Introdução}

A tuberculose bovina ou tuberculose zoonótica é uma zoonose de evolução crônica causada por uma bactéria, o Mycobacterium bovis. Esta bactéria é do gênero Mycobacterium que se apresentam como bastonetes Gram positivos, aeróbios estritos, imóveis, não esporulados e álcool-ácido resistentes na coloração Ziehl-Neelsen (Ruggiero, et al., 2007; Caetano, 2014; Oliveira, 2015). Além disso, o agente etiológico da TB é muito resistente e em condições favoráveis pode sobreviver longos períodos no ambiente (Caetano, 2014).

A doença tem distribuição mundial, e mesmo com a enfermidade pertencendo à lista de doenças de notificação obrigatória da Organização Mundial de Saúde Animal (OIE), o que reforça a importância da TB como um grave problema de saúde pública (Riet-Correa, et al., 2007; Rocha, et al., 2012).

A tuberculose zoonótica provocada pelo M. bovis já foi relatada em uma infinidade de espécies de hospedeiros, dentre eles, mamíferos domésticos e silvestres, herbívoros (bovinos, alces, bisontes, búfalos, cervos, ovinos, caprinos, elefantes, rinocerontes, aves, seres humanos, dentre outros (Sabedot, et al., 2009; OIE, 2020). Dentre as espécies domésticas, os bovinos e suínos são os principais hospedeiros (Almeida, Soares \& Araújo, 2004).

Os bovinos afetados, mesmo antes de desenvolver lesões teciduais, podem disseminar o patógeno, por descarga nasal, leite, fezes, urina, secreções nasal, vaginal, uterina e sêmen (Almeida, Soares \& Araújo, 2004).

A tuberculose bovina promove significativas perdas econômicas na bovinocultura pois causa aumento da mortalidade animal, redução de produtividade de carne e leite, condenação de carcaça em frigoríficos e restrições nas exportações (Rocha, et al., 2012; FAO, OMS \& OIE, 2017).

A transmissão da tuberculose zoonótica se dá por via direta aerógena, por meio da inalação do patógeno, ou via indireta, pelo consumo de leite contaminado cru que não recebeu nenhum tipo de tratamento térmico. A transmissão via 
consumo de carne contaminada crua ou mal-cozida também pode ocorrer, mas é menos recorrente, pois a presença do M. bovis na musculatura é rara, porém, este tipo de transmissão não deve ser descartado principalmente devido ao grande número de abates clandestinos ou e abates realizados em matadouros municipais que não executam as normas de inspeção sanitária (Dias, 2012; WHO, OIE \& FAO, 2017).

Segundo a Organização Mundial de Saúde (OMS), estima-se o surgimento de 10 milhões de casos novos de tuberculose no mundo, com óbitos de 1 milhão de pessoas. Em 2016, foram registrados no mundo cerca de 147.000 novos casos de tuberculose zoonótica, com 12.500 mortes (OMS, 2016; OIE, 2020; Olea-Popelka, et al., 2016). Mesmo com número elevado de casos, a tuberculose é considerada doença negligenciada, e estima-se que em alguns países $10 \%$ dos casos de tuberculose humana são originados de tuberculose bovina (OIE, 2020).

Geralmente, o cuidado na diferenciação do agente etiológico promotor do caso de tuberculose humana não é realizado (Davidson, et al., 2017). Desta forma, possivelmente existe subnotificação dos casos de tuberculose zoonótica, pois estes casos são notificados apenas como tuberculose humana.

A partir de 2006, a OMS estabeleceu como paradigma visando o controle das zoonoses, a necessidade de cooperação entre a medicina veterinária e a medicina humana, pois esta interrelação colabora positivamente na elaboração de pesquisas no campo de epidemiologia, controle, diagnóstico, prevenção e vigilância das enfermidades que acometem os seres vivos de modo geral (Silva, et al., 2018). Neste contexto a presente pesquisa tem por objetivo descrever a epidemiologia dos casos de tuberculose bovina na América do Sul, visando enfatizar a importância das medidas de controle e prevenção da doença.

\section{Metodologia}

Para esta pesquisa foi realizado um estudo transversal, descritivo, retrospectivo e qualiquantitativo (Pereira, et al., 2018) que utilizou como unidades de análise países localizados na América do Sul, com ênfase o Brasil.

A amostra foi delimitada ao período de 2012 a 2018, e os dados foram coletados a partir das informações disponíveis em banco de dados oficiais da Organização Internacional de Epizootias (OIE) e do Ministério da Agricultura, Pecuária e Abastecimento (MAPA). As informações coletadas das notificações foram: ano, semestre, país, unidades da federação brasileira e número total de casos notificados e número total de focos.

Os dados referentes ao efetivo de rebanho bovino do Brasil, foi obtido por meio de consulta ao site do Instituto Brasileiro de Geografia e Estatística (IBGE), no campo Pesquisa de Pecuária Municipal, e referente ao número de estabelecimentos, os dados utilizados são do último censo agropecuário realizado em 2017.

Para o cálculo das prevalências, utilizou-se as seguintes equações matemáticas:

$$
\begin{gathered}
\text { Coef. Prevalência }=\frac{\text { número casos notificados }}{\text { rebanho estimado }} \times 10.000 \\
\text { Coef.Prevalência focos }=\frac{\text { número de propriedades notificados }}{\text { total de propriedades }} \times 10.000
\end{gathered}
$$

Após coleta dos dados e cálculo dos índices, os resultados foram analisados por meio de estatística descritiva simples e apresentados em formato de gráficos e tabelas.

\section{Resultados e Discussão}

A tuberculose bovina é uma doença extremamente importante por se tratar de uma zoonose com ampla distribuição, que provoca elevado número de mortes em seres humanos e que além disso, promove perdas econômicas na pecuária, desde 
alterações de desenvolvimento, produtividade e morte do animal até o impacto negativo no comércio de seus produtos.

De acordo com dados disponibilizados pela OIE, nos países que compõe a América do Sul, com exceção do Brasil, os casos notificados de 2012 a 2018, estão descritos na Figura 1.

Figura 1 - Quantitativo de casos notificados de tuberculose bovina em países da América do Sul, exceto o Brasil, de 2012 a 2018.

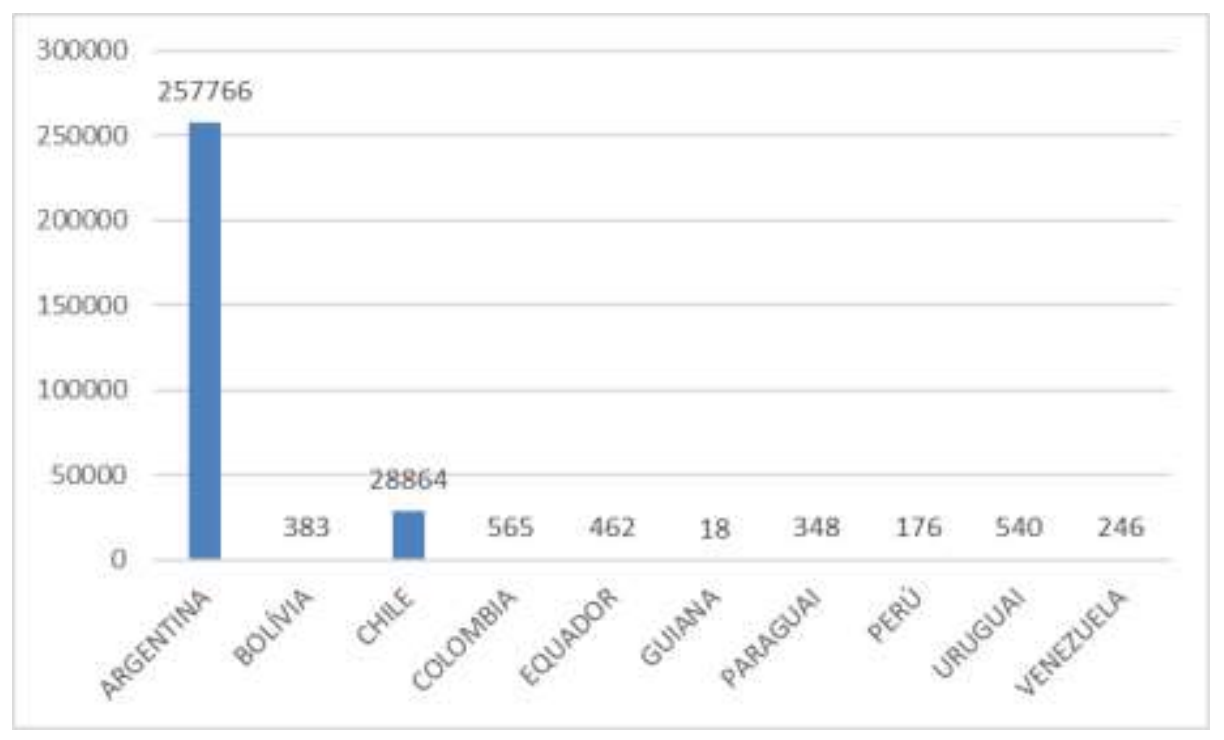

Fonte: Autores.

De acordo com a Figura 1, os países que apresentaram maior número de casos de tuberculose bovina no período estudado foram Argentina e Chile. A Argentina teve grande destaque em casos notificados quando comparado aos outros países, pois apresentou 8,9 vezes mais notificações que o segundo colocado, o Chile.

De acordo com a OIE, a tuberculose bovina está disseminada por todo o mundo, e apresenta maior prevalência em grande parte da África, partes da Ásia, Oceania e na maioria dos países sul-americanos (OIE, 2018).

O número elevado de notificações na Argentina e Chile pode indicar excelência no sistema de vigilância da tuberculose bovina no país, assim como, a baixa notificação realizada pelos demais países pode indicar a ocorrência de subnotificações e ausência da realização de diagnóstico. Porém, em ambas as situações, ações de controle e prevenção à doença devem ser tomadas com foco em sua erradicação.

Dados da OIE revelaram que em 2016, foram registrados no mundo, cerca de 147 mil novos casos de tuberculose zoonótica e 12.500 óbitos, porém, a notificação incorreta dos dados pode levar a subestimação da capacidade zoonótica por M. bovis e a potencial queda nas notificações (OIE, 2020; Olea-Popelka, et al., 2016).

Dentre os anos estudados, a distribuição dos casos notificados de tuberculose bovina em países da América do Sul, exceto o Brasil, está discriminada na Figura 2. 
Figura 2 - Distribuição, por ano de ocorrência, dos casos notificados de Tuberculose Bovina em países da América do Sul, exceto o Brasil, de 2012 a 2018.

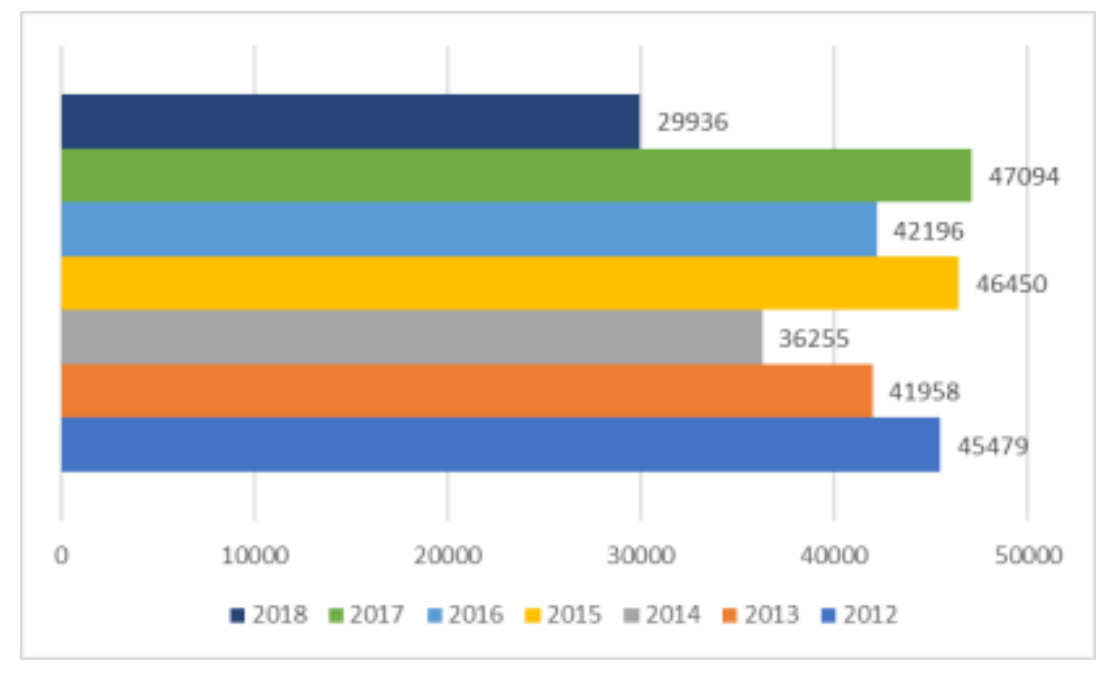

Fonte: Autores.

Dentre os anos estudados, 2017 apresentou o maior número de casos notificados, perfazendo 16,3\%, seguido pelo ano de 2015 , com $16,1 \%$ e 2012 com $15,7 \%$.

A distribuição dos casos em países da América do Sul, exceto o Brasil, com relação aos semestres em que foram realizadas as notificações demonstrou que $52 \%$ foram realizadas no primeiro semestre, ou seja, dentre os meses de janeiro a junho, $48 \%$ no segundo semestre, entre julho e dezembro.

Com relação ao Brasil, os dados disponibilizados pelo MAPA compreenderam os anos de 2012 a 2019. Neste período, o efetivo de rebanho bovino, de acordo com as unidades da federação, compõe a Tabela 1. 
Research, Society and Development, v. 10, n. 9, e8610917936, 2021

(CC BY 4.0) | ISSN 2525-3409 | DOI: http://dx.doi.org/10.33448/rsd-v10i9.17936

Tabela 1 - Efetivo de rebanho bovino dos estados brasileiros de 2012 a 2019.

\begin{tabular}{|c|c|c|c|c|c|c|c|c|}
\hline & 2012 & 2013 & 2014 & 2015 & 2016 & 2017 & 2018 & 2019 \\
\hline $\mathbf{A C}$ & 2634467 & 2697489 & 2799673 & 2916207 & 2998969 & 2895028 & 3303633 & 3509682 \\
\hline $\mathbf{A L}$ & 1221266 & 1251723 & 1253121 & 1255696 & 1189985 & 1208404 & 1247619 & 1233394 \\
\hline AP & 142825 & 154967 & 167529 & 89682 & 76379 & 66921 & 55422 & 54296 \\
\hline $\mathbf{A M}$ & 1445739 & 1470537 & 1405208 & 1293325 & 1315821 & 1343574 & 1376210 & 1455842 \\
\hline BA & 10250975 & 10828409 & 10824134 & 10758372 & 10363291 & 10037814 & 9923931 & 10214863 \\
\hline $\mathrm{CE}$ & 2714713 & 2591067 & 2597139 & 2516197 & 2426408 & 2287400 & 2403371 & 2479289 \\
\hline DF & 100069 & 101452 & 100056 & 96576 & 96265 & 93141 & 90199 & 84425 \\
\hline $\mathbf{E S}$ & 2285345 & 2313445 & 2295624 & 2223531 & 2044771 & 1937604 & 1976903 & 2006027 \\
\hline GO & 22045776 & 21580398 & 21538072 & 21887720 & 22919070 & 22835005 & 22651910 & 22785151 \\
\hline MA & 7490942 & 7611324 & 7758352 & 7643128 & 7653870 & 7687695 & 7793180 & 8008643 \\
\hline MT & 28740802 & 28395205 & 28592183 & 29364042 & 30296096 & 29725378 & 30199598 & 31973856 \\
\hline MS & 21498382 & 21047274 & 21003830 & 21357398 & 21800990 & 21474693 & 20896700 & 19407908 \\
\hline MG & 23965914 & 24201256 & 23707042 & 23768959 & 23637803 & 21971713 & 21810311 & 22020979 \\
\hline PA & 18605051 & 19165028 & 19911217 & 20271618 & 20476783 & 20585367 & 20628651 & 20881204 \\
\hline PB & 967067 & 1048824 & 1145943 & 1170803 & 1187981 & 1202781 & 1240004 & 1293769 \\
\hline PR & 9413937 & 9395313 & 9181577 & 9314908 & 9487999 & 9370139 & 9275271 & 8971675 \\
\hline PE & 1895642 & 1823230 & 1920075 & 1948357 & 1895185 & 1804876 & 1862181 & 1933900 \\
\hline PI & 1689926 & 1666107 & 1660099 & 1649549 & 1639856 & 1625006 & 1464196 & 1447817 \\
\hline RJ & 2197615 & 2339978 & 2379648 & 2351451 & 2409718 & 2531239 & 2552587 & 2533164 \\
\hline $\mathbf{R N}$ & 858211 & 914777 & 972816 & 918952 & 840847 & 870000 & 863284 & 929451 \\
\hline $\mathbf{R S}$ & 14140654 & 14037367 & 13956953 & 13737316 & 13590282 & 13353122 & 12550379 & 11968216 \\
\hline RO & 12218437 & 12329971 & 12744326 & 13397970 & 13682200 & 14091378 & 14367161 & 14349219 \\
\hline RR & 686491 & 747045 & 735962 & 794783 & 780877 & 787318 & 817198 & 879007 \\
\hline SC & 4072960 & 4201561 & 4285931 & 4382299 & 4499505 & 4302861 & 4296052 & 4452571 \\
\hline SP & 10757383 & 10486750 & 10148423 & 10468135 & 11031408 & 11109523 & 10771635 & 10486465 \\
\hline SE & 1156157 & 1223215 & 1218972 & 1231130 & 1196248 & 1067121 & 1039346 & 1052263 \\
\hline TO & 8082336 & 8140580 & 8062227 & 8412404 & 8652161 & 8738477 & 8352513 & 8480724 \\
\hline
\end{tabular}

Fonte: IBGE (2021).

Os estados que apresentaram efetivo médio de rebanho bovino maior durante o período de estudo, foram Mato Grosso, Minas Gerais, Goiás e Mato Grosso do Sul. Em Mato Grosso, Goiás e Mato Grosso do Sul, a atividade pecuária predominante é a bovinocultura de corte e em Minas Gerais a bovinocultura leiteira.

Dentre o período avaliado, foram notificados 36.986 casos de tuberculose bovina no Brasil. A distribuição destas notificações de acordo com a unidade da federação de ocorrência está descrita na Figura 3. 
Figura 3 - Distribuição das notificações de casos de Tuberculose Bovina no Brasil, de acordo com as unidades da federação, de 2012 a 2019.

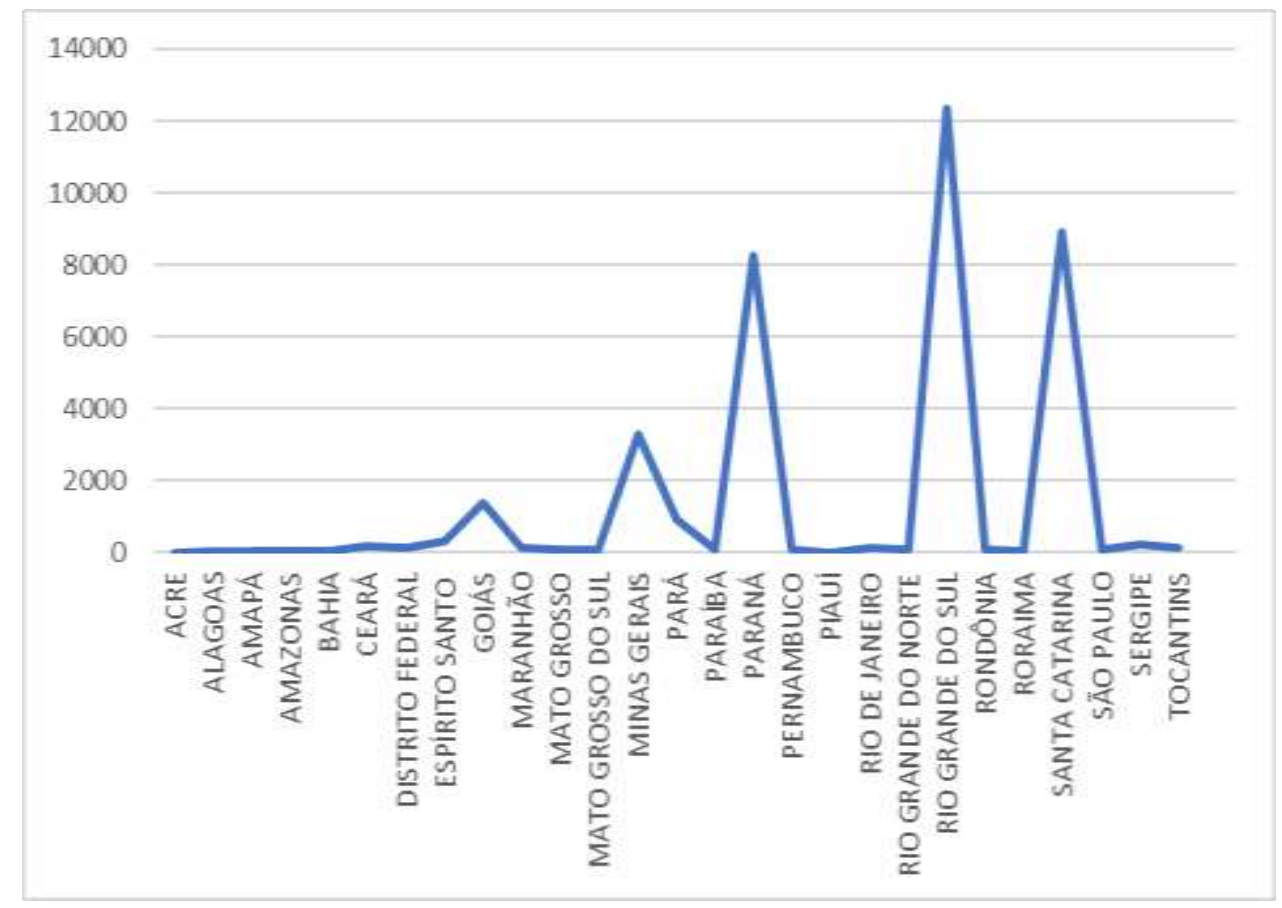

Fonte: Autores.

Na Figura 3 nota-se que os estados que mais notificaram casos de tuberculose bovina no período foram Rio Grande do Sul (12.390 notificações), Santa Catarina (8.898 notificações), Paraná (8.262 notificações) e Minas Gerais (3.274 notificações). Estes estados são extremamente representativos para a bovinocultura leiteira.

Estudos comprovaram que rebanhos leiteiros tem maior tendência a adquirir a doença, por isso, propriedades com sistema de produção mista (leite e corte) ou de produção leiteira geralmente apresentam maior prevalência da doença (Dias, et al., 2016; Veloso, et al., 2016), o que pode justificar o elevado número de notificações nos estados citados acima.

De acordo com Barbieri, et al., (2016), propriedades leiteiras com sistema de criação intensivo possuem alto risco para ocorrência de tuberculose no rebanho. Fatores relacionados a densidade populacional, a entrada de novos animais, permanência longa do animal na propriedade, falta de cuidados veterinários e contato com espécies com potencial para transmissão, fazem com que os rebanhos leiteiros sejam mais predispostos a possuir animais positivos.

Dentre os estados que se destacam na pecuária de corte, o que mais notificou casos de tuberculose bovina foi o estado de Goiás (1.390 notificações). Este número de notificações representa apenas 11,2\% das notificações realizadas pelo estado do Rio Grande do Sul (local que realizou o maior número de notificações).

A produção de bovinos de corte na região Centro Oeste do Brasil caracteriza-se por criação extensiva e a pasto, o que pode justificar a baixa prevalência de animais positivos encontrados nesta região (Guedes, et al., 2016; Néspoli, et al., 2016; Ribeiro, et al., 2016; Rocha, et al., 2016).

Ao analisar a taxa de prevalência da doença de acordo com o efetivo de rebanho de cada estado, notou-se taxa mais elevada em Santa Catarina (0,21\%), Distrito Federal (0,11\%), Rio Grande do Sul (0,09\%), e Paraná (0,09\%).

Fatores relacionados a subnotificação de casos, e a baixa realização de testes diagnósticos influenciam diretamente a taxa de prevalência da doença, desta forma, a prevalência encontrada pode estar subestimada se estes fatores estiverem presentes nos estados brasileiros.

Algumas pesquisas determinaram taxas de prevalência da tuberculose bovina em algumas regiões brasileiras. Costa, 
(2012) citou 0,9 a 2,9\% de prevalência nas principais regiões econômicas do estado da Bahia; Bahiense, et al., (2016) relataram valores médios de 0,21\% no estado da Bahia; Barbieri, et al., (2016) em Minas Gerais detectaram 0,56\% de prevalência; Silva, et al., (2016) detectaram 0,42\% prevalência no Paraná; Veloso, et al., (2016), em Santa Catarina, descreveram prevalência da doença abaixo de 1\%; Lima, et al., (2016) detectaram 0,62\% em Pernambuco; e Vendrame, et al., (2016), 0,12\% de prevalência da doença em Rondônia.

Ao avaliar a quantidade total de casos notificados de tuberculose bovina de acordo com o ano de ocorrência, os dados estão discriminados na Figura 4.

Figura 4 - Número total de casos notificados de Tuberculose Bovina no Brasil, de 2012 a 2019.

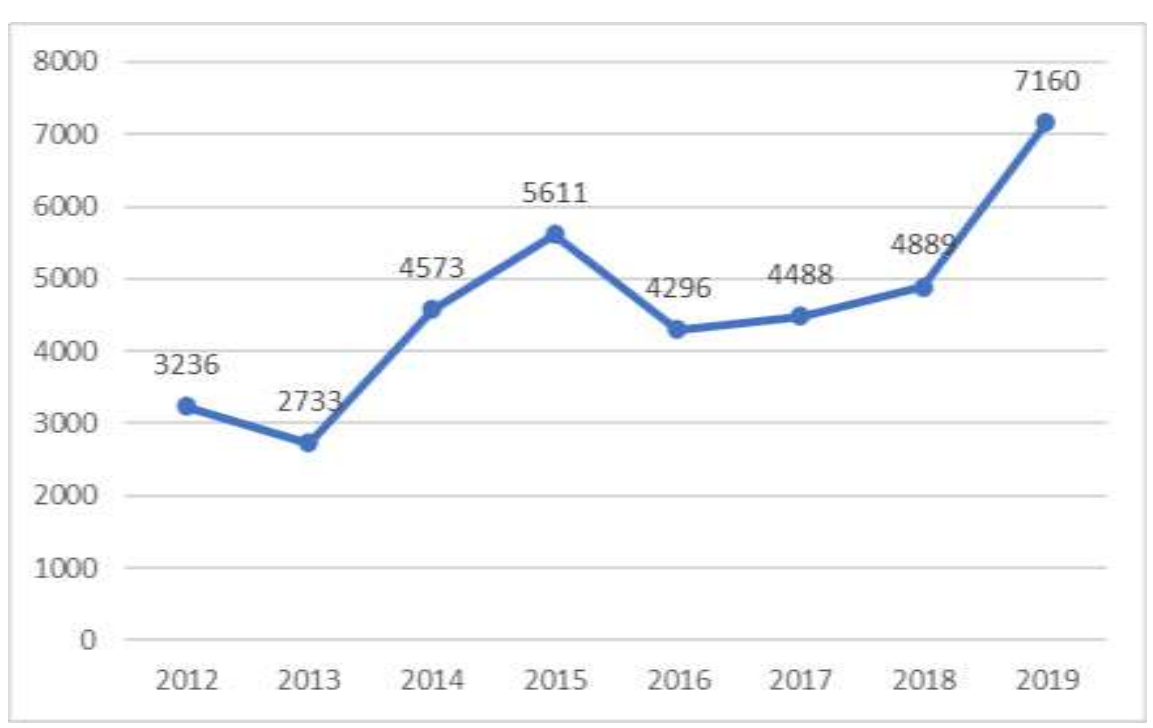

Fonte: Autores.

Com relação ao total de notificação dos casos de tuberculose bovina no Brasil, destaca-se o ano de 2015, pois ocorreu um pico de notificações. Em 2016, o número de notificações caiu, mas nos anos seguintes voltou a subir, apresentando ápice no ano de 2019. Este dado é importante pois o aumento do número de casos notificados pode indicar que a vigilância da tuberculose bovina está ativa e atuando de forma eficaz no país.

Em contrapartida, o aumento de casos pode indicar também falhas nas medidas de controle e prevenção da doença no país, o que requer melhorias referentes a realização de diagnóstico precoce, controle eficiente da doença e implementação de medidas de biossegurança nos rebanhos.

De acordo com os dados obtidos, o Brasil apresentou prevalência média no período da pesquisa de $0,02 \%$, enquanto a Argentina apresentou prevalência média de 0,46\%, e o Chile 0,96\%. Estudo realizado por Azamora Filho (2014) revelou 1,3\% de prevalência para tuberculose bovina no Brasil, dado este com valor acima do encontrado nesta pesquisa.

Ações devem ser intensificadas com objetivo de baixar a prevalência da doença no mundo, dentre elas a realização de testes de diagnóstico e abate dos animais positivos que já demonstraram ser eficientes e promoveram a erradicação da doença em países como a França e a Alemanha, e baixou a prevalência da doença a níveis residuais na Bulgária e Romênia (Cunha, et al., 2019).

Ao analisar a época do ano que mais ocorreu notificações, observou-se que dentre os 36.986 casos, $48 \%$ foram relatados entre os meses de janeiro e junho e, $52 \%$ entre julho e dezembro, o que não apresentou diferença, ou seja, as notificações ocorrem de forma homogênea durante todo os meses do ano.

Com relação ao número de focos relacionados ao número de casos de tuberculose bovina notificados, os dados estão 
descritos na Figura 5.

Figura 5 - Número de focos de tuberculose bovina relacionados ao número de casos notificados no Brasil, de 2012 a 2019.

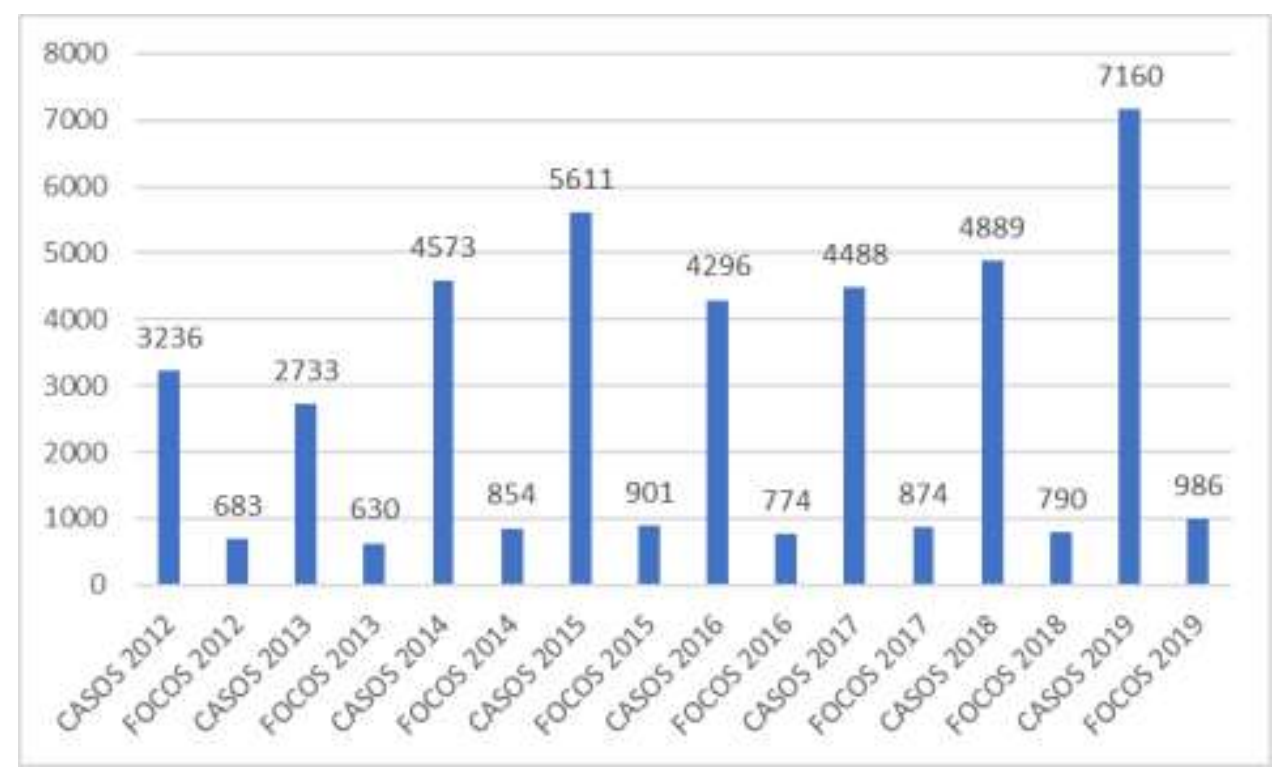

Fonte: Autores.

O número de focos de uma determinada afecção pode indicar o quanto ela está disseminada em uma determinada região. Nesta pesquisa, os anos de 2015 e 2019 apresentaram maior número de focos de tuberculose bovina no Brasil. Com relação ao coeficiente de prevalência dos focos, este está descrito na Tabela 2. 
Tabela 2 - Coeficiente de prevalência de focos de tuberculose bovina de acordo com as Unidades da Federação, de 2012 a 2019.

\begin{tabular}{|c|c|c|c|}
\hline UF & ESTABELECIMENTOS & FOCOS & PREVALÊNCIA (\%) \\
\hline ACRE & 22649 & 4 & 0,02 \\
\hline ALAGOAS & 42300 & 21 & 0,05 \\
\hline AMAPÁ & 1709 & 3 & 0,18 \\
\hline AMAZONAS & 14612 & 17 & 0,12 \\
\hline BAHIA & 297894 & 11 & 0,00 \\
\hline CEARÁ & 117550 & 51 & 0,04 \\
\hline DISTRITO FEDERAL & 1468 & 19 & 1,29 \\
\hline ESPÍRITO SANTO & 33128 & 79 & 0,24 \\
\hline GOIÁS & 102551 & 238 & 0,23 \\
\hline MARANHÃO & 91296 & 47 & 0,05 \\
\hline MATO GROSSO & 73925 & 19 & 0,03 \\
\hline MATO GROSSO DO SUL & 40281 & 60 & 0,15 \\
\hline MINAS GERAIS & 342771 & 631 & 0,18 \\
\hline PARANÁ & 164137 & 2212 & 1,35 \\
\hline PARAÍBA & 82761 & 50 & 0,06 \\
\hline PARÁ & 97769 & 113 & 0,12 \\
\hline PERNAMBUCO & 107939 & 35 & 0,03 \\
\hline PIAUÍ & 108843 & 0 & 0,00 \\
\hline RIO GRANDE DO NORTE & 38137 & 31 & 0,08 \\
\hline RIO GRANDE DO SUL & 239779 & 1629 & 0,68 \\
\hline RIO DE JANEIRO & 20705 & 28 & 0,14 \\
\hline RONDÔNIA & 57934 & 67 & 0,12 \\
\hline RORAIMA & 6903 & 4 & 0,06 \\
\hline SANTA CATARINA & 111007 & 982 & 0,88 \\
\hline SERGIPE & 36185 & 33 & 0,09 \\
\hline SÃO PAULO & 52373 & 26 & 0,05 \\
\hline TOCANTINS & 48549 & 82 & 0,17 \\
\hline
\end{tabular}

Fonte: Autores.

As informações contidas na Tabela 2 demonstram que os estados de Paraná (1,35\%), Distrito Federal (1,29\%) e Santa Catarina $(0,88 \%)$ apresentaram as maiores taxas de prevalência de focos de tuberculose bovina.

Barbiere, et al., (2016) realizaram um estudo em Minas Gerais e encontraram prevalência de focos de tuberculose bovina de 4,25\%. No estado na Bahia, Bahiense, et al., (2016) relataram 1,6\% de prevalência de focos, e no Espírito Santo, Galvis, et al., (2016) obtiveram uma prevalência de 7,6\% das propriedades com bovinos reagentes.

No Rio Grande do Sul, estudo apontou 2,8\% de prevalência de foco. No Paraná o valor obtido foi de 2,15\%, Santa Catarina a prevalência está abaixo de 1\%, Pernambuco, 2,87\% e Rondônia, 2,33\% (Lima, et al., 2016; Queiroz, et al., 2016; Silva, et al., 2016; Vendrame, et al., 2016; Veloso, et al., 2016).

Prevalência de foco acima de $1 \%$ indica disseminação da doença na localidade estudada, e nestes casos, existe a necessidade de implementação de ações imediatas e efetivas dos órgãos de defesa sanitária animal e humana com foco em controle e prevenção da doença.

Devido à complexidade da epidemiologia da tuberculose bovina, com sua gama elevada de hospedeiros, domésticos ou silvestres que possuem relação estreita com o ser humano, fica clara a necessidade da doença ser abordada de uma forma revolucionária e dentro do contexto de Saúde Única, para que as características de cada região seja compreendida e as melhores formas de prevenção e controle da doença sejam tomadas. 


\section{Conclusão}

Os países da América do Sul, inclusive o Brasil, possuem baixa taxa de prevalência de tuberculose bovina, porém estratégias relacionadas a vigilância da doença devem ser tomadas com foco no diagnóstico e correta destinação dos animais positivos. Para isso, deve-se investir em programas de educação em saúde voltados aos produtores rurais, para que entendam a importância da realização do diagnóstico laboratorial nos animais de seu rebanho e naqueles frutos de aquisição, antes da entrada na propriedade.

Devido ao fato do desconhecimento da população com relação a doença, uma grande gama de indivíduos está exposta ao risco de contágio, desta forma, estudos epidemiológicos com base em registros oficiais e in loco devem ser realizados para proporcionar o desenvolvimento de medidas de controle e prevenção pertinentes, aliando ações no âmbito de Saúde Única, para diminuir o risco de ocorrência da tuberculose zoonótica.

\section{Agradecimentos}

Universidade Brasil, Campus Fernandópolis, São Paulo

\section{Referências}

Almeida, R. F. C., Soares, C. O. \& Araújo, F. F. (2004). Brucelose e Tuberculose Bovina: Epidemiologia, controle e diagnóstico. Embrapa.

Alzamora Filho, F., Vasconcellos, S. E. G., Gomes, H. M., Cavalcante, M. P., Suffys, P. N. \& Costa, J. N. (2014). Múltiplas estirpes de isolados de Mycobacterium bovis identificados por tipagem molecular em bovinos abatidos em matadouros frigoríficos. Pesquisa Veterinária Brasileira, 34 (2), $103-108$. 10.1590/S0100-736X2014000200001.

Bahiense, L., Ávila, L. N., Bavia, M. E., Amaku, M., Dias, R. A., Grisi-Filho, J. H. H., Ferreira, F., Telles, E. O., Gonçalves, V. S. P., Heinemann, M. B. \& Ferreira Neto, J. S. (2016). Prevalence and risk factors for bovine tuberculosis in the State of Bahia, Brazil. Semina: Ciências Agrárias, 37(5), 3549-3559. $10.5433 / 1679-0359.2016 v 37 n 5 \operatorname{Supl} 2 \mathrm{p} 3549$.

Barbieri, J. M., Oliveira, L. F., Dorneles, E. M. S., Mota, A. L., Gonçalves, V. S., Maluf, P. P., Ferreira Neto, J. S., Ferreira, F., Dias, R. A., Telles, E. O., Grisi-Filho, J. H. H., Heinemann, M. B., Amaku, M. \& Lage, A. P. (2016). Epidemiological status of bovine tuberculosis in the state of Minas Gerais, Brazil, 2013. Semina: Ciencias Agrarias, 37(5), 3531-3548. 10.5433/1679-0359.2016v37n5Supl2p3531.

Caetano, P. M. (2014). Clínica de espécies pecuárias e equinos: Avaliação epidemiológica da tuberculose bovina em espécies de caça maior, nas regiões do Alto Alentejo e Beira Interior Sul. Dissertação (Mestrado - Medicina Veterinária) - Mestrado Integrado em Medicina Veterinária. Évora, Portugal.

Costa. L. B. (2012). Caracterização da tuberculose bovina em regiões de relevância econômica no Estado da Bahia. Tese (Doutorado em Ciências Animal) Universidade Federal da Bahia. Salvador, Bahia, Brasil.

Cunha, M. V., Pereira, A. C., Reis, A. C. \& Ramos, B. (2019). Tuberculose animal: diagnóstico, epidemiologia, investigação e controlo. Vida Rural, 38-44. https://www.researchgate.net/publication/337915556_Tuberculose_animal_diagnostico_epidemiologia_investigacao_e_controlo.

Davidson, J. A., Loutet, M. G., O’connor, C., Kearns, C., Smith, R. M. M., Lalor, M. K., Thomas, H. L., Abubakar, I. \& Zenner, D. (2017). Epidemiology of Mycobacterium bovis Disease in Humans in England, Wales, and Northern Ireland, 2002-2014. Emerging Infectious Diseases, 23(3), 2017. 10.3201/eid2303.161408.

Dias, I. C. (2012). Prevenção de zoonoses ocupacionais em abatedouros de bovinos. Revista Eletrônica de Extensão da URI, 8(15), 89-96. http://www2.reitoria.uri.br/ vivencias/Numero_015/artigos/pdf/Artigo_07.pdf.

Dias, R. A., Ulloa-Stanojlovic, F. M., Belchior, A. P. C., Ferreira, R. S., Gonçalves, R. C., Aguiar, R. S. C. B., Sousa, P. R., Santos, A. M. A., Amaku, M.; Ferreira, F., Telles, E. O., Grisi-Filho, J. H. H., Golçalves, V. S. P., Heinemann, M. B. \& Ferreira Neto, J. S. (2016). Prevalence and risk factors for bovine tuberculosis in the state of São Paulo, Brazil. Semina: Ciências Agrárias, 37(5), 3673-3684. 10.5433/1679-0359.2016v37n5Supl2p3673.

FAO., OMS., OIE. (2021). Hoja de ruta contra la tuberculosis zoonótica. http://www.who.int/iris/handle/10665/259231.

Galvis, J. O. A., Grisi-Filho, J. H. H., Costa, D., Said, A. L. P. R., Amaku, M., Dias, R. A., Ferreira, F., Gonçalves, V. S. P., Heinemann, M. B., Telles, E. O. \& Ferreira Neto, J. S. (2016). Epidemiologic characterization of bovine tuberculosis in the state of Espírito Santo, Brazil. Semina: Ciências Agrárias, 37(5), 3567-3578. 10.5433/1679-0359.2016v37n5Supl2p3567.

Guedes, I. B., Bottene, I. F. N., Monteiro, L. A. R. C. M., Leal Filho, J. M., Heinemann, M. B., Amaku, M., Grisi-Filho, J. H. H., Dias, R. A., Ferreira, F., Telles, E. O., Salvador, V. G. P. \& Ferreira Neto, J. S. (2016). Prevalence and risk factors for bovine tuberculosis in the state of Mato Grosso do Sul, Brazil. Semina: Ciências Agrárias, 37(5), 3579-3588. 10.5433/1679-0359.2016v37n5Supl2p3579.

Lima, P. R. B., Nascimento, D. L., Almeida, E. C., Pontual, K. A. Q., Amaku, M., Dias, R. A., Ferreira, F., Gonçalves, V. S. P., Telles, E. O., Grisi-Filho, J. H. H., Heinemann, M. B., Silva, J. C. R. \& Ferreira Neto, J. S. (2016). Epidemiological situation of bovine tuberculosis in the state of Pernambuco, Brazil. Semina: Ciências Agrárias, 37(5), 3601-3610. 10.5433/1679-0359.2016v37n5Supl2p3601. 
Néspoli, J. M. B., Negreiros, R. L., Amaku, M., Dias, R. A., Ferreira, F., Telles, E. O., Heinemann, M. B., Grisi-Filho, H. H. H., Golçalves, V. S. P. \& Ferreira Neto, J. S. (2016). Epidemiological situation of bovine tuberculosis in the state of Mato Grosso, Brazil. Semina: Ciências Agrárias, 37(5), 3589-3600. 10.5433/1679-0359.2016v37n5Supl2p3589.

OIE. Organização Mundial de Saúde Animal. (2017). Roadmap for zoonotic tuberculosis. https://www.oie.int/fileadmin/Home/eng/Our_scientific_e xpertise/docs/pdf/Tuberculosis/Roadmap_zoonotic_TB.pdf.

OIE. Organização Mundial de Saúde Animal. (2018). Mapa de distribuição de doenças. https://www.oie.int/wahis_2/public/wahid.php/Diseaseinforma tion/Diseasedis tributionmap.

OIE. Organização Mundial de Saúde Animal. (2020). Bovine Tuberculosis. http://www.oie.int/fileadmin/Home/eng/Media_Center/docs/pdf/Dis ease_cards/BOVINE-TB-EN.pdf.

Olea-Popelka, F., Muwonge, A., Perera, A., Dean, A. S., Mumford, E., Erlacher-Vindel, E., Forcella, S., Silk, B. J., Ditiu, L., El Idrissi, A., Raviglione, M., Cosivi, O., Lobue, P. \& Fujiwara, P. L. (2016). Zoonotic tuberculosis in human beings caused by Mycobacterium bovis - a call for action. The Lancet, 17(1), 21-25. 10.1016/S1473-3099(16)30139-6.

Oliveira, R. M. (2015). Estudo da Tuberculose como Doença de Declaração Obrigatória em Abates Normais de Bovinos. Mestrado (Medicina Veterinária) Mestrado Integrado em Medicina Veterinária. Lisboa, Portugal.

Pereira, A. S., Shitsuka, D. M., Parreira, F. J., Shitsuka, R. (2018). Metodologia da pesquisa científica. UFSM.

Ruggiero, A. P., Ikuno, A. A., Ferreira, V. C. A. \& Roxo, E. (2007). Tuberculose bovina: alternativas para o diagnóstico. Arquivos do Instituto Biológico, 74(1), 55-65. http://www.biologico.sp.gov.br/uploads/docs/arq/V74_1/a49f0f8e-f4a4-47ef-9aba-c628606281b2.pdf.

Queiroz, M. R., Groff, A. C. M., Silva, N. S., Grisi-Filho, J. H. H., Amaku, M., Dias, R. A., Telles, E. O., Heinemann, M. B., Ferreira Neto, J. S., Gonçalves, V. S. P. \& Ferreira, E. (2016). Epidemiological status of bovine tuberculosis in the state of Rio Grande do Sul, Brazil. Semina: Ciências Agrárias, 37(5), 36473658. 10.5433/1679-0359.2016v37n5Supl2p3647.

Ribeiro, L. A., Gonçalves, V. S. P., Francisco, P. F. C., Mota, A. L. A. A., Nascimento, G. T., Licurgo, J. B., Ferreira, F., Grisi-Filho, J. H. H., Ferrira Neto, J. S., Amaku, M., Dias, R. A., Telles, E. O., Heinemann, M. B. \& Borges, J. R. J. (2016). Epidemiological statu s of bovine tuberculosis in the Federal District of Brazil. Semina: Ciências Agrárias, 37(5), 3561-3566. 10.5433/1679-0359.2016v37n5Sup12p3561.

Riet-Correa, F., Schild, A. L., Mendez, M. D. C. \& Lemos, R. A. A. (2007). Doenças de Ruminantes e Equídeos. 3. Brasil, São Paulo: Fernovi Editora.

Rocha, V.C.F., Figueiredo, S.C., Elias, A.O., Leão, D.A.S. \& Neto, J.S.F. Mycobacterium bovis como agente causal da tuberculose humana. (2012). Revista de Educação Continuada em Medicina Veterinária e Zootecnia, 10(2), 22-31. 10.36440/recmvz.v10i2/3.252.

Rocha, W. V., Jayme, V. S., Mota, A. L. A. A., Brito, W. M. E. D., Pires, G. R. C., Ferreira Neto, J. S., Grisi-Filho, J. H. H., Dias, R. A., Amaku, M., Telles, E. O., Ferreira, F., Heinemann, M. B. \& Gonçalves, V. S. P. (2016). Prevalence and herd level risk factors of bovine tuberculosis in the State of Goiás, Brazil. Semina: Ciências Agrárias, 37(5), 3625-3628. 10.5433/1679-0359.2016v37n5Sup12p3625.

Sabedot, M. A., Boetcher, A. V., Pozza, M. S. S., Busanello, M. \& Mangoni, J. (2009). Ocorrência de tuberculose e brucelose em rebanhos da região sudoeste do Paraná. Revista Científica Eletrônica de Medicina e Veterinária, 7(12). http://faef.revista.inf.br/imagens_arquivos/arquivos _destaque/TWIFqvD9Wrivjwa_2013-6-18-15-57-10.pdf.

Silva, M. C. P., Gonçalves, V. S. P., Mota, A. L. A. A., Koloda, M., Ferreira Neto, J. S., Grisi-Filho, J. H. H., Dias, R. A., Amaku, M., Telles, E. O., Ferreira, F., Heinemann, M. B., Alfieri, A. A. \& Muller, E. E. (2016). Prevalence and herd level risk factors for bovine tuberculosis in the state of Paraná, Brazil. Semina: Ciências Agrárias, 37(5), 3611-3624. 10.5433/1679-0359.2016v37n5Sup12p3611.

Silva, M. R., Rocha, A. S., Araujo, F. R., Fonseca-Junior, A. A., Alencar, A. P., Suffys, P. N., Costa, R. R., Moreira, M. A. S. \& Guimaraes, M. D. C. (2018). Risk factors for human Mycobacterium bovis infections in a urban area of Brazil. Memórias do Instituto Oswaldo Cruz, 113(8),1- 6. 10.1590/007402760170445 .

Vendrame, F. B., Amaku, M., Ferreira, F., Telles, E. O., Grisi-Filho, J. H. H., Golçalves, V. S. P., Heinemann, M. B., Ferreira Neto, J. S. \& Dias, R. A. (2016). Epidemiologic characterization of bovine tuberculosis in the State of Rondônia, Brazil. Semina: Ciências Agrárias, 37(5), 3639-3646. 10.5433/16790359.2016v37n5Supl2p3639.

Veloso, F. P., Baumgerten, K. D., Mota, A. L., Ferreira, F., Neto, J. S., Grisi-Filho, J. H. H., Dias, R. A., Amaku, M., Telles, E. O. \& Gonçalves, V. S. P. (2016). Prevalence and herd-level risk factors of bovine tuberculosis in the State of Santa Catarina. Semina: Ciências Agrárias, 37(5), 3659-3672. 10.5433/1679-0359.2016v37n5Supl2p3659. 